\title{
Évolution quinquennale de la régénération protégée lors de récoltes par abattage manuel dans des sapinières
}

\author{
par Pierre Pominville
}

En 1985, une expérience a été entreprise en Gaspésie, au Québec, dans le but d'évaluer l'effet de la coupe avec protection de la régénération dans plusieurs types de peuplements et pour différents procédés de récolte. Cet article traite de l'abattage manuel avec débusquage à câble dans les sapinières. Des mesures ont été prises avant, immédiatement après, de même que 3 ans et 5 ans après la coupe dans cinq paires de blocs de 2 ha chacun afin de comparer la méthode traditionnelle (témoins) et celle des sentiers espacés avec abattage directionnel (protégés).

Immédiatement après la coupe, on obtient une baisse plus faible du coefficient de distribution des résineux dans le bloc protégé de chaque paire. Cinq ans plus tard, cette différence est maintenue et le gain moyen qui en découle est de $25 \%$. La régénération est aussi légèrement plus haute dans les blocs protégés. Ceci semble attribuable au fait que la méthode étudiée permet d'atténuer l'envahissement par les arbustes et les herbacées après la coupe.
In 1985, an experiment was undertaken in the Gaspé peninsula, in Québec, in different stand types to assess the feasibility of preserving advance growth by changing logging methods. This article deals with manual felling combined with wheeled cable skidders in balsam fir stands. Regeneration was assessed before, immediately after, and 3 and 5 years after logging in five 2-ha pairs of blocks, in order to compare the effect of traditional harvesting (control) and a method using directional felling and spaced skidding trails (preserved).

The use of directional felling and spaced skidding trails resulted in a decreased loss of advance growth. Five years after logging, this loss in softwood stocking for the preserved blocks was still lower in each of the five pairs; the difference averaged $25 \%$. Moreover, the regeneration height in the preserved blocks was slightly greater, which seems to indicate a lower level of invasion by brush and herbaceous vegetation after logging as a result of this method.

\section{Introduction}

La régénération préétablie est abondante dans toutes les zones écologiques et tous les types de peuplements résineux mûrs du Québec, sauf ceux de pin gris (Doucet 1988). Une importante proportion de cette régénération est toutefois détruite lors de la récolte de bois (Webber et al. 1968). Or, depuis 1987, la Loi sur les forêts oblige la remise en production des parterres de coupe (Québec 1991). Pour ce faire, d'autres moyens que la plantation doivent être envisagés. En effet, selon Tremblay et al. (1987), s'il fallait renouveler toutes les surfaces récoltées par cette approche, les coûts atteindraient 260 millions \$ par an. Par ailleurs, les coûts de protection de la régénération sont minimes selon une étude du ministère de l'Énergie et des Ressources du Québec (MER) qui englobe des essais sur plus de 800 ha, entre 1979 et 1988 . On y conclut que seule la formation de la maind'oeuvre entraîne des coûts supplémentaires temporaires ${ }^{1}$.

Les procédés utilisant les débusqueuses sont les plus destructeurs, occasionnant des baisses du coefficient de distribution de la régénération de $30 \%$ et plus (Ruel 1987). Or, ces débusqueuses sont présentes sur neuf chantiers de coupe sur dix ${ }^{2}$. L'utilisation de transporteurs est une façon de pallier à ce problème (Godin 1986). D'autres mesures permettent aussi de préserver la régénération préétablie: coupe sur couvert de neige (Frank et Putman 1972; Ruel 1987; 1989a), pneus à haute flottaison (Heidersdorf et Ryans 1986), sentiers espacés avec abattage directionnel (Canuel 1987).

Ces méthodes sont particulièrement prometteuses dans les sapinières, où le coefficient de distribution des résineux affiche une moyenne de $90 \%$ avant la coupe (Doucet 1988). Malgré tout, on estime à $20 \%$ les sapinières mal régénérées après la coupe (Ruel 1989b). De plus, l'absence de mesures de protection peut entraîner d'importantes répercussions sur

$\overline{\text { Gouvernement du Québec, ministère des Forêts, Service de la recherche }}$ appliquée, 2700, rue Einstein, Sainte-Foy, Québec, G1P 3W8.

la composition et la structure de la régénération. L'envahissement par les arbustes et les herbacées, provoqué par la perturbation du sol, en est un exemple (Marks 1974; Wall 1983).

Cet article compare la régénération observée 5 ans après la coupe dans des sapinières récoltées par le procédé traditionnel d'abattage manuel suivi d'un débusquage à câble (témoins) à celle obtenue avec la méthode des sentiers espacés avec abattage directionnel (protégés).

\section{Méthode}

\section{Description des secteurs expérimentaux}

Une expérience a été entreprise en 1983 dans différents peuplements résineux et mixtes du Québec. Parmi ceux-ci, des sapinières de l'unité de gestion de la Gaspésie (région administrative 11) ont été sélectionnées pour la présente étude. Le MER (Hardy 1988) y établissait en 1985 un dispositif de 10 blocs-échantillons carrés d'environ 2 ha chacun. Le tableau 1 présente les principales caractéristiques des peuplements échantillonnés. Aucune attention particulière n'a été portée à la régénération préétablie dans les blocs témoins. Dans les blocs protégés, les opérateurs utilisaient toute la longueur du câble de la débusqueuse afin d'obtenir le maximum d'espacement entre les sentiers. De plus, les directions des tiges abattues permettaient de réduire leur surface de balayage lors de la tirée vers le sentier de débusquage. La récolte des blocs d'une même paire était effectuée par la même équipe de travail. La plupart des ouvriers expérimentaient la méthode des sentiers espacés avec abattage directionnel pour la première fois. La distance entre les sentiers primaires a varié entre 20 et $40 \mathrm{~m}$.

Tous les blocs avaient une régénération préétablie de hauteur moyenne inférieure à $30 \mathrm{~cm}$. Le sapin baumier

\footnotetext{
${ }^{1}$ Gilles Rhéaume, Service des techniques d'intervention forestière du ministère des Forêts du Québec, communication personnelle.

${ }^{2}$ Bruno Canuel, Service des techniques d'intervention forestière du ministère des Forêts du Québec, communication personnelle.
} 


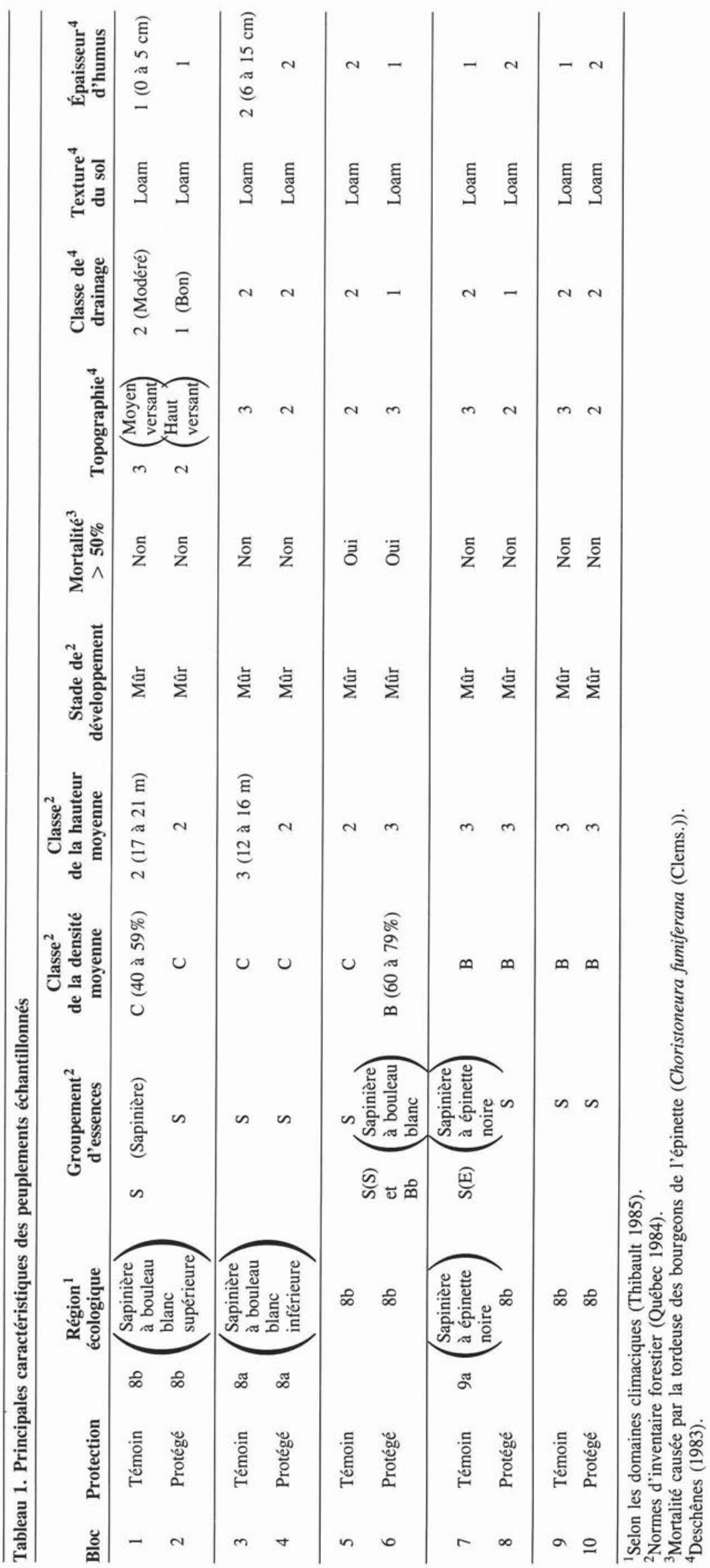


(Abies balsamea [L.] Mill) était l'espèce la mieux représentée, suivie du bouleau blanc (Betula papyrifera Marsh.) et des épinettes (Picea spp.). Les blocs témoins et protégés avaient des coefficients de distribution équivalents avant la coupe (figure 1). Les coefficients de distribution des résineux étaient représentatifs des sapinières des régions écologiques 8a, 8b et 9a de Thibault (1985) (Ruel 1989b).

\section{Évolution des coefficients de distribution}

Des inventaires de régénération ont été effectuées avant, immédiatement après, de même que 3 et 5 ans après la coupe. Des placettes échantillons de $4 \mathrm{~m}^{2}$ ont été distribuées suivant un sondage systématique avec points de départ aléatoires. Les virées étaient équidistantes, parallèles entre elles (Deschênes 1983) et formaient un angle variable avec les sentiers de débusquage. Le nombre minimal, moyen et maximal de placettes par bloc fut de 56,89 et 118 . Les virées et points de départ utilisés avant la coupe ont de nouveau été employés lors des inventaires subséquents.

Une évaluation globale de la situation 5 ans après la coupe a d'abord été faite. À cette fin, une comparaison des coefficients de distribution (CDR) a été effectuée à l'intérieur de chaque paire de blocs. Par la suite, les relations des CDR des blocs témoins et protégés 5 ans après la coupe en fonction d'avant la coupe ont été analysées. Des tests du T ont servi aux différentes comparaisons. L'évolution des principales espèces a aussi été étudiée afin d'évaluer la stabilité des résultats obtenus et les tendances à venir.

\section{Régénération et compétition}

Afin de comparer la compétition dans les blocs témoins et protégés, des tests du T ont été effectués sur les variables suivantes: coefficient de distribution de la tige dominante de régénération, hauteur moyenne de la régénération, hauteur moyenne de la compétition et couverture de la compétition.

Le coefficient de distribution de la tige dominante de régénération ne tient compte que de la plus haute tige présente dans une placette donnée. Pour les compétiteurs, la hauteur moyenne a remplacé celle de la plus haute tige puisqu'une seule tige d'épilobe (Epilobium spp.) ou de framboisier (Rubus idaeus L.) (principaux compétiteurs dans les blocs étudiés) ne suffit pas à compétitionner le sapin (Hatcher 1964a; Wall 1983; Ruel 1992).

\section{Résultats et discussion}

\section{Évolution des coefficients de distribution}

Cinq ans après la coupe, la baisse du coefficient de distribution (CDR) des résineux est plus faible dans les blocs protégés (voir la variation au tableau $2 \mathrm{a}$ ). Le gain qui en découle par rapport aux témoins (calculé par paire de blocs) s'est maintenu durant toute la période d'observation. Malgré tout, 5 ans après la coupe, trois des cinq blocs protégés (quatre sur cinq dans le cas des témoins) ont un CDR des résineux inférieur au seuil de $60 \%$. Ce seuil correspond au niveau nécessaire à l'atteinte de la production maximale d'une station donnée (Pominville et Doucet 1992).

Cinq après la coupe, le sapin donne des résultats équivalents à ceux de l'ensemble des résineux. Son gain moyen $(\bar{x})$ est nettement significatif $(\bar{x}=24,4$; écart-type $(s)=8,6 ; t=$ $6,4 ;[P>t]=0,0031)$. L'effet de la coupe avec protection de la régénération sur les épinettes semble toutefois beaucoup plus imprévisible $(\bar{x}=-0,6 ; s=13,1 ; t=0,1$; $[P>t]=0,92)$. Ce fait est sans doute dû à une plus grande variabilité pour cette espèce, occasionnée par des valeurs plus faibles avant la coupe. Par ailleurs, la baisse moyenne du CDR du bouleau blanc a été plus forte dans les blocs protégés.

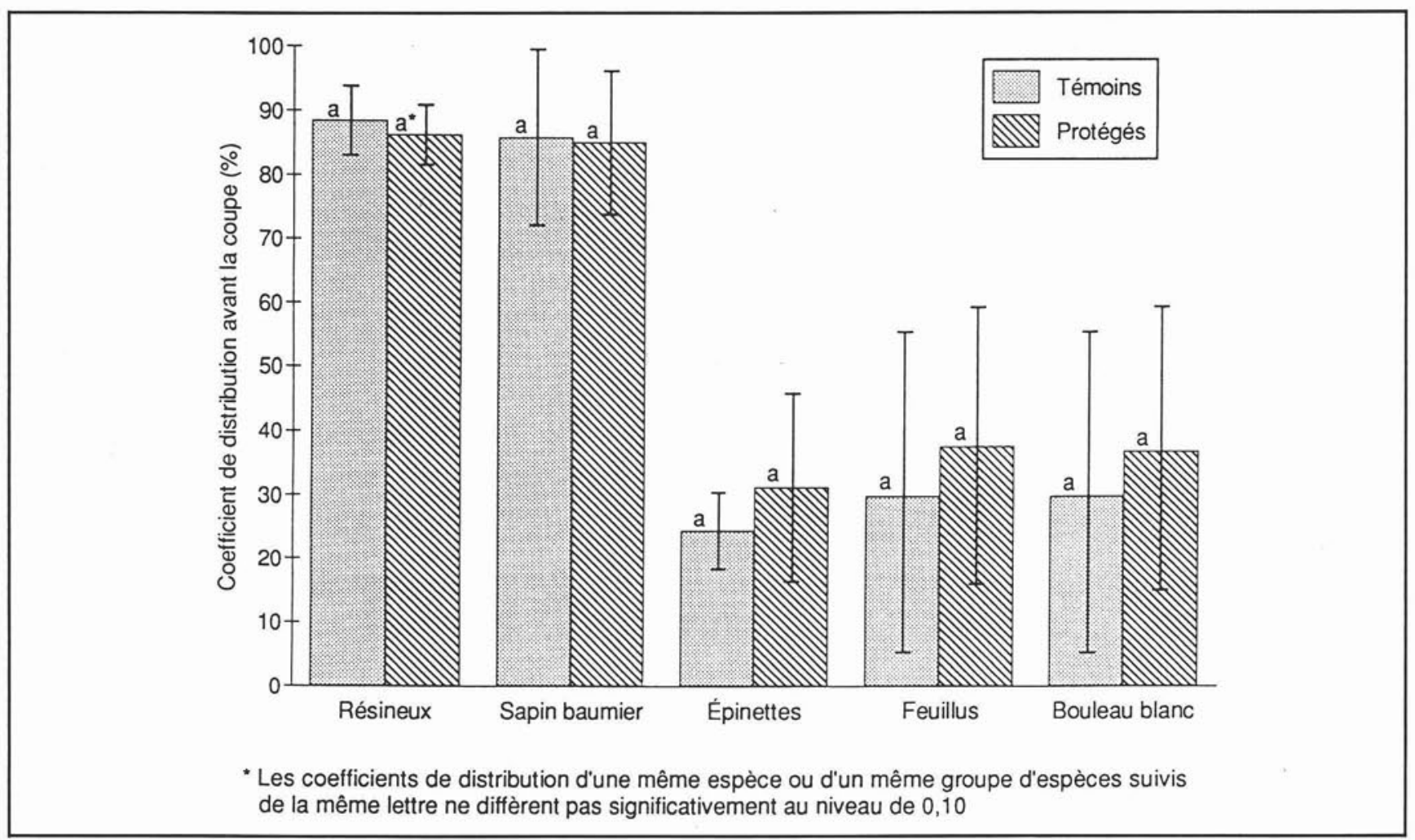

Figure 1. Coefficients de distribution des blocs témoins et protégés avant la coupe. 
Tableau 2. Évolution après la coupe des coefficients de distribution et gain obtenu dans les blocs protégés

(a) résineux

\begin{tabular}{|c|c|c|c|c|c|c|c|c|c|}
\hline \multirow[b]{2}{*}{ Bloc } & \multirow[b]{2}{*}{ Méthode } & \multirow[b]{2}{*}{$\begin{array}{c}\text { Mortalité } \\
>\mathbf{5 0 \%}\end{array}$} & \multirow[b]{2}{*}{ Protection } & \multicolumn{5}{|c|}{ Coefficient de distribution (CDRR) (\%) } & \multirow{2}{*}{$\begin{array}{c}\text { Gain }(\%) \\
\begin{array}{c}\text { Protégé } \\
\text { moins } \\
\text { témoin }\end{array}\end{array}$} \\
\hline & & & & $\begin{array}{l}\text { Avant } \\
\text { coupe } \\
\text { (1) }\end{array}$ & $\begin{array}{l}\text { Après } \\
\text { coupe }\end{array}$ & $\begin{array}{l}3 \text { ans } \\
\text { après }\end{array}$ & $\begin{array}{l}5 \text { ans } \\
\text { après } \\
\text { (2) }\end{array}$ & $\begin{array}{c}\text { Variation } \\
(2)-(1)\end{array}$ & \\
\hline $\begin{array}{l}1 \\
2\end{array}$ & $\begin{array}{l}\text { Tronc } \\
\text { entier }\end{array}$ & Non & $\begin{array}{l}\text { Témoin } \\
\text { Protégé }\end{array}$ & $\begin{array}{l}96 \\
90\end{array}$ & $\begin{array}{l}60 \\
74\end{array}$ & $\begin{array}{l}34 \\
53\end{array}$ & $\begin{array}{l}44 \\
47\end{array}$ & $\begin{array}{l}-52 \\
-43\end{array}$ & 9 \\
\hline $\begin{array}{l}3 \\
4\end{array}$ & $\begin{array}{l}\text { Tronc } \\
\text { entier }\end{array}$ & Non & $\begin{array}{l}\text { Témoin } \\
\text { Protégé }\end{array}$ & $\begin{array}{l}77 \\
80\end{array}$ & $\begin{array}{l}21 \\
50\end{array}$ & $\begin{array}{l}38 \\
52\end{array}$ & $\begin{array}{l}35 \\
56\end{array}$ & $\begin{array}{l}-42 \\
-24\end{array}$ & 18 \\
\hline $\begin{array}{l}5 \\
6\end{array}$ & $\begin{array}{l}\text { Tronc } \\
\text { entier }\end{array}$ & Oui & $\begin{array}{l}\text { Témoin } \\
\text { Protégé }\end{array}$ & $\begin{array}{l}74 \\
72\end{array}$ & $\begin{array}{l}43 \\
63\end{array}$ & $\begin{array}{r}9 \\
49\end{array}$ & $\begin{array}{l}18 \\
54\end{array}$ & $\begin{array}{l}-56 \\
-18\end{array}$ & 38 \\
\hline $\begin{array}{l}7 \\
8\end{array}$ & $\begin{array}{l}\text { Arbre } \\
\text { entier }\end{array}$ & Non & $\begin{array}{l}\text { Témoin } \\
\text { Protégé }\end{array}$ & $\begin{array}{r}100 \\
99\end{array}$ & $\begin{array}{l}69 \\
91\end{array}$ & $\begin{array}{l}66 \\
93\end{array}$ & $\begin{array}{l}69 \\
92\end{array}$ & $\begin{array}{r}-31 \\
-8\end{array}$ & 23 \\
\hline $\begin{array}{r}9 \\
10\end{array}$ & $\begin{array}{l}\text { Arbre } \\
\text { entier }\end{array}$ & Non & $\begin{array}{l}\text { Témoin } \\
\text { Protégé }\end{array}$ & $\begin{array}{l}95 \\
90\end{array}$ & $\begin{array}{l}54 \\
78\end{array}$ & $\begin{array}{l}51 \\
77\end{array}$ & $\begin{array}{l}50 \\
81\end{array}$ & $\begin{array}{r}-45 \\
-9\end{array}$ & 36 \\
\hline \multicolumn{3}{|c|}{ MOYENNE } & $\begin{array}{l}\text { Témoins } \\
\text { Protégés }\end{array}$ & $\begin{array}{l}88,4 \\
86,2\end{array}$ & $\begin{array}{l}49,4 \\
71,2\end{array}$ & $\begin{array}{l}39,6 \\
64,8\end{array}$ & $\begin{array}{l}43,2 \\
66,0\end{array}$ & $\begin{array}{l}-45,2 \\
-20,2\end{array}$ & 24,8 \\
\hline
\end{tabular}

(b) feuillus

\begin{tabular}{|c|c|c|c|c|c|c|c|c|c|}
\hline \multirow[b]{2}{*}{ Bloc } & \multirow[b]{2}{*}{ Méthode } & \multirow[b]{2}{*}{$\begin{array}{c}\text { Mortalité } \\
>\mathbf{5 0 \%}\end{array}$} & \multirow[b]{2}{*}{ Protection } & \multicolumn{5}{|c|}{ Coefficient de distribution (CDRF) (\%) } & \multirow{2}{*}{$\begin{array}{c}\text { Gain }(\%) \\
\text { Protégé } \\
\text { moins } \\
\text { témoin }\end{array}$} \\
\hline & & & & $\begin{array}{c}\text { Avant } \\
\text { coupe } \\
\text { (1) }\end{array}$ & $\begin{array}{l}\text { Après } \\
\text { coupe }\end{array}$ & $\begin{array}{l}3 \text { ans } \\
\text { après }\end{array}$ & $\begin{array}{l}5 \text { ans } \\
\text { après } \\
\text { (2) }\end{array}$ & $\begin{array}{l}\text { Variation } \\
(2)-(1)\end{array}$ & \\
\hline 1 & Tronc & \multirow{2}{*}{ Non } & Témoin & 30 & 12 & 44 & 37 & +7 & \multirow{2}{*}{0} \\
\hline 2 & entier & & Protégé & 29 & 15 & 52 & 36 & +7 & \\
\hline 3 & Tronc & \multirow{2}{*}{ Non } & Témoin & 19 & 2 & 55 & 45 & +26 & \multirow{2}{*}{-30} \\
\hline 4 & entier & & Protégé & 27 & 17 & 31 & 23 & -4 & \\
\hline 5 & Tronc & \multirow{2}{*}{ Oui } & Témoin & 14 & 4 & 23 & 25 & +11 & \multirow{2}{*}{-3} \\
\hline 6 & entier & & Protégé & 36 & 13 & 40 & 44 & +8 & \\
\hline 7 & Arbre & \multirow{2}{*}{ Non } & Témoin & 14 & 8 & 59 & 63 & +49 & \multirow{2}{*}{-31} \\
\hline 8 & entier & & Protégé & 75 & 33 & 92 & 93 & +18 & \\
\hline 9 & Arbre & \multirow{2}{*}{ Non } & Témoin & 72 & 20 & 70 & 67 & -5 & \multirow{2}{*}{+17} \\
\hline 10 & entier & & Protégé & 21 & 11 & 31 & 33 & +12 & \\
\hline \multirow{2}{*}{\multicolumn{3}{|c|}{ MOYENNE }} & Témoins & 29,8 & 9,2 & 50,2 & 47,4 & $+17,6$ & \multirow{2}{*}{$-9,4$} \\
\hline & & & Protégés & 37,6 & 17,8 & 49,2 & 45,8 & $+8,2$ & \\
\hline
\end{tabular}

*CDRR: coefficient de distribution de la régénération des résineux.

**CDRF: coefficient de distribution de la régénération des feuillus.

La perte qui en découle n'a cependant pas été significative, compte tenu de la forte variabilité observée avant la coupe $(\bar{x}=-21,3 ; s=30,3 ; t=-1,5 ;[P>t=0,1967)$. Ceci s'est réflété sur l'ensemble des feuillus (voir le gain au tableau $2 \mathrm{~b}$ ) qui affichent aussi une perte non significative $([P>t]=0,3677)$ pour les blocs protégés. D'autre part, les gains obtenus dans les blocs récoltés par troncs entiers et par arbres entiers ne sont pas significativement différents $([P>t]=0,5353)$.

Une relation significative $([P>F]=0,0398)$ du CDR 5 ans après la coupe en fonction du CDR avant la coupe a été obtenue pour les résineux dans les blocs témoins (figure 2a). Les blocs protégés, pour leur part, ont montré plus de variabilité. Toutefois, tous les points qui représentent ces derniers se trouvent au-dessus de la droite des témoins. Le gain des protégés sur les témoins semble donc indépendant du coefficient des résineux avant la coupe."

Chez les feuillus (figure $2 \mathrm{~b}$ ) ce sont les blocs protégés qui ont donné une relation significative $([P>F]=0,0051)$.
Le CDR des feuillus est presque toujours plus élevé 5 ans après la coupe qu'avant celle-ci. Seuls les blocs 4 et 9 (tableau $2 \mathrm{~b}$ ) ont montré une légère diminution, de 4 et $5 \%$ respectivement. De plus, pour les valeurs du CDR des feuillus les plus susceptibles d'être rencontrées avant la coupe (moins de 40 p. 100), on note une augmentation plus prononcée dans les secteurs témoins.

Ce phénomène semble logique. En effet, la perturbation du sol, plus importante dans les blocs non protégés, provoque de nouvelles germinations du bouleau blanc (Marquis et al. 1969; Safford et al. 1990). Dans l'échantillon étudié, les feuillus sont essentiellement représentés par cette espèce. Ainsi, les blocs témoins ayant un faible CDR des feuillus avant la coupe compenseront facilement par de nouvelles germinations les pertes causées lors de la coupe. Par contre, ceux qui ont un CDR des feuillus élevé avant la coupe subiront une baisse plus considérable donc, plus difficile à compenser. La perturbation étant moins importante dans les blocs protégés, les germinations après la coupe y sont plus rares; c'est 
pourquoi le CDR des feuillus observé avant la coupe y augmente peu par la suite.

Â ce stade, il y a lieu de se demander si l'ouverture du couvert par la tordeuse des bourgeons de l'épinette (TBE) a eu un effet sur le CDR du bouleau blanc avant la coupe. Si oui, cela entraînerait un effet indirect sur le CDR des feuillus 5 ans après la coupe. Le bouleau blanc, intolérant à l'ombre, peut s'établir abondamment sous couvert mais survit rarement plus de cinq ans en l'absence d'ouverture (Côté et Bélanger 1991; Perkins et al. 1992). On peut donc s'attendre à ce que la TBE, en créant des ouvertures, favorise la survie et, par conséquent, l'augmentation progressive du CDR des feuillus avant la coupe. Plusieurs auteurs ont observé une plus forte proportion de feuillus dans les peuplements ouverts par la TBE (Fye et Thomas 1963; Hatcher 1964b, Ohman et Ream 1971; Mott 1974; Potvin 1980; Maclean 1984). Ainsi, le CDR des feuillus avant la coupe pourrait être à peu près proportionnel au degré d'ouverture du couvert par la TBE. Cependant, une telle tendance n'a pas été observée dans l'échantillon étudié. Ainsi, les deux seuls blocs qui montraient une mortalité supérieure à $50 \%$ avaient un CDR des feuillus inférieur à $40 \%$ avant la coupe alors que deux autres moins touchés par la TBE avaient des CDR des feuillus de 72 et $75 \%$.

L'influence de la TBE pourrait, dans une certaine mesure, avoir été contrebalancée par d'autres facteurs (type et épaisseur d'humus et de litière, drainage, compétition interspécifique). L'impact de ces facteurs sur l'établissement du bouleau blanc avant la coupe aurait été plus déterminant que la quantité de lumière. Côté et Bélanger (1991) ont observé que le bouleau blanc était très sensible aux variations du milieu de croissance. Par ailleurs, Perkins et al. (1992) n'ont pas obtenu de différences significatives de densité à l'intérieur de microsites équivalents dans des trouées et sous couvert. En somme, dans l'échantillon étudié, la mortalité occasionnée par la TBE ne semble pas avoir influencé de façon déterminante le CDR des feuillus 5 ans après la coupe.

$\mathrm{La}$ figure $3 \mathrm{a}$ montre que la diminution du CDR des résineux s'est surtout produite au moment de la coupe ou au cours de l'année qui a suivi. Cette diminution, sans doute attribuable aux blessures d'exploitation et à l'incapacité d'adaptation de certains semis face à une intensité lumineuse accrue après la coupe (Ruel 1990), a été plus faible au cours des deux années suivantes. Le CDR s'est stabilisé par la suite.

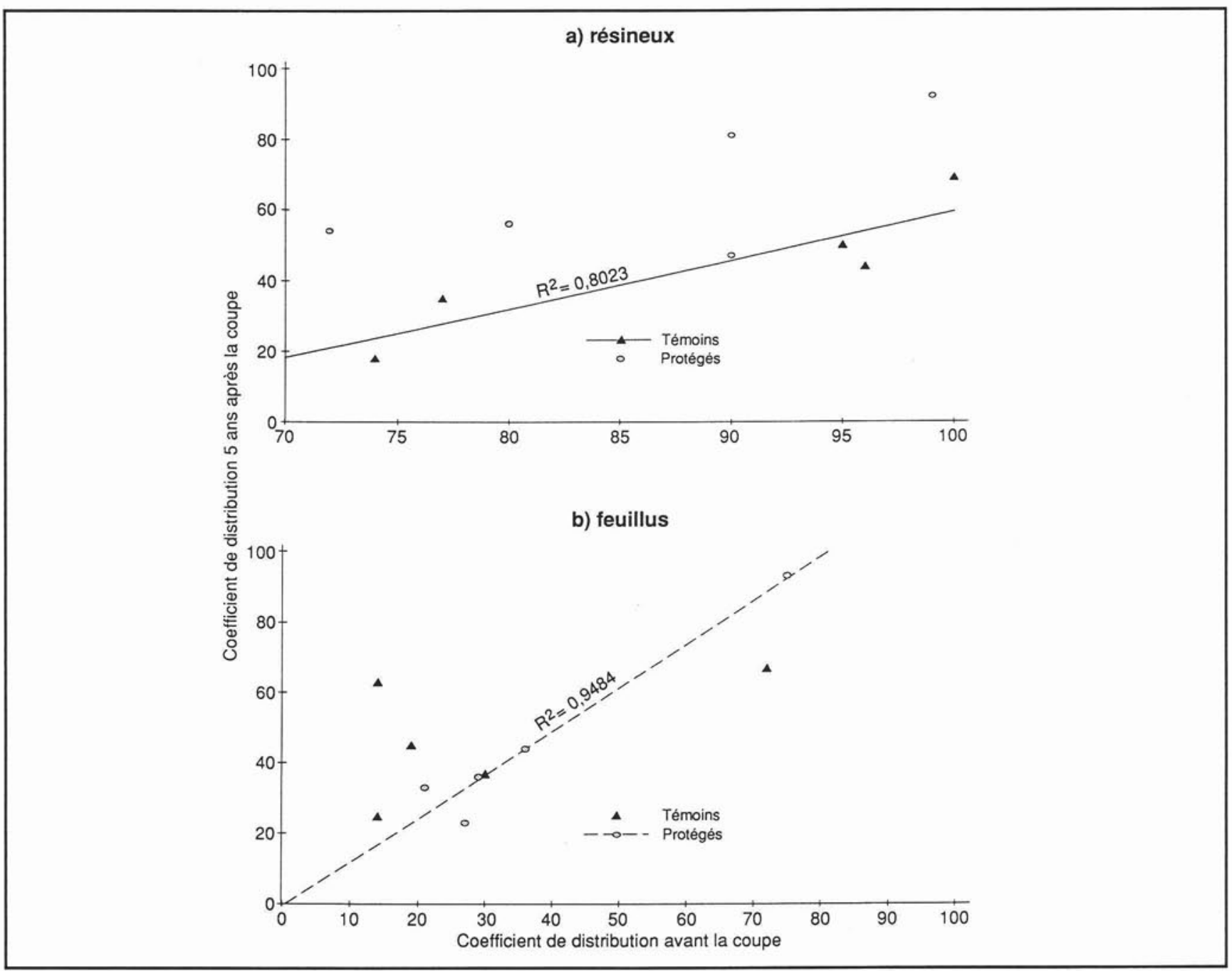

Figure 2. Coefficients de distribution des résineux et des feuillus 5 ans après la coupe en fonction d'avant la coupe dans les blocs témoins et protégés récoltés par abattage manuel et débusquage à câble. 
a) résineux

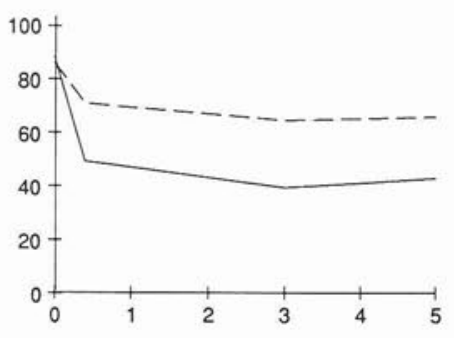

b) sapin baumier

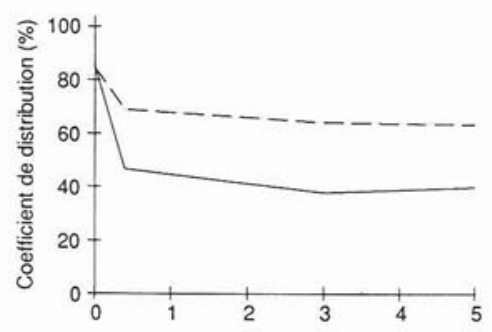

c) épinettes

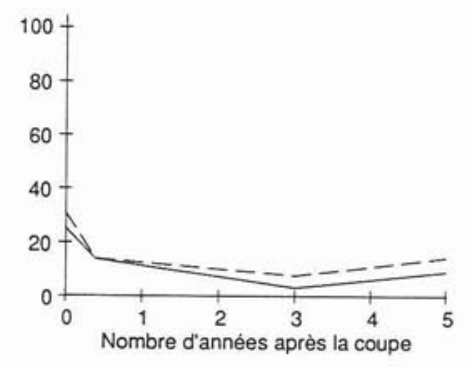

d) feuillus

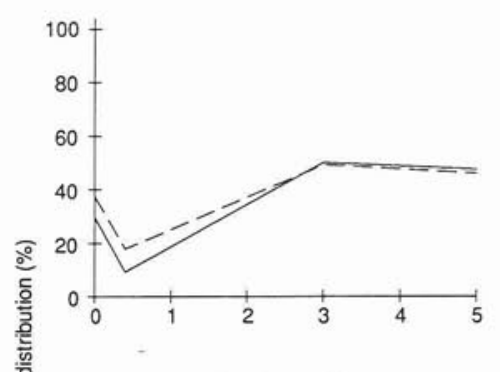

e) bouleau blanc

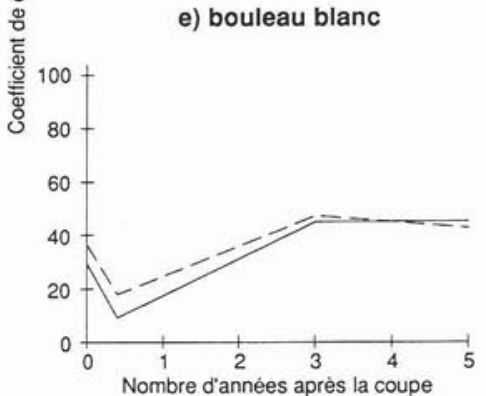

Figure 3. Évolution après la coupe des coefficients de distribution des blocs témoins et protégés récoltés par abattage manuel et débusquage à câble.

Il pourrait même augmenter à l'avenir, si l'accroissement du CDR des épinettes (figure 3c) se poursuivait.

Le phénomène a déjà été observé dans les pessières noires. Huot et Ruel (1990) obtiennent un accroissement du CDR des résineux 5 ans après une récolte par abattage manuel et débusquage. Deschênes (1991) a observé, sur des sites humides, une augmentation du CDR des résineux de $30 \%$ échelonnée sur une période de 14 ans après la coupe. Il attribue ce fait au passage dans la classe supérieure des tiges non comptées lors du premier inventaire (hauteur inférieure à $5 \mathrm{~cm}$ ) et aux nouvelles germinations qui s'observent même en l'absence de semenciers ${ }^{1}$. La capacité de germination des semences d'épinette noire (Picea mariana [Mill.] B.S.P.) dépasse rarement 1 an, une fois en contact avec le sol (Fraser 1976). Ces nouvelles germinations proviendraient donc en bonne partie de la libération progressive des semences contenues dans les branches laissées sur le parterre de coupe. Une partie de ces semences conservent la capacité de germer pendant plusieurs années (Schoenike et Hansen 1954).

L'évolution des feuillus, essentiellement constituées par le bouleau blanc, diffère considérablement de celle des

${ }^{1}$ Claude Deschênes, Service de la recherche appliquée du ministère des Forêts du Québec, communication personnelle. résineux. Le CDR de cette espèce de lumière augmente après la coupe pendant environ 3 ans, puis semble se stabiliser (figure 3e). Cette stabilisation est sans doute due, entre autres, à la courte durée de la capacité de germination de ses graines, soit environ 1 an (Marquis et al. 1969, Safford et al. 1990). La compétition interspécifique a peut-être aussi un rôle à jouer.

\section{Régénération et compétition}

Le coefficient de distribution des tiges dominantes résineuses des blocs protégés surpasse celui des témoins de $15 \%$. Toutefois, cette différence n'est pas significative compte tenu de la variabilité observée (figure 4). Chez les témoins, la majeure partie des surfaces est dominée par les arbustes et les herbacées, la dominance résineuse étant plutôt marginale. Ce fait pourrait avoir une incidence sur la croissance. Ainsi, Lanteigne et Methven (1986) ont observé un retard de croissance de l'ordre de 30 ans dans les sapinières dominées par le cerisier de Pennsylvanie (Prunus pensylvanica L.f.) après la coupe. Dans le cas présent cependant, le framboisier étant le principal concurrent, les répercussions devraient être moins accentuées (Ruel 1992).

Bien qu'arbustes et herbacées aient des hauteurs équivalentes dans les deux groupes de blocs, il n'en demeure 


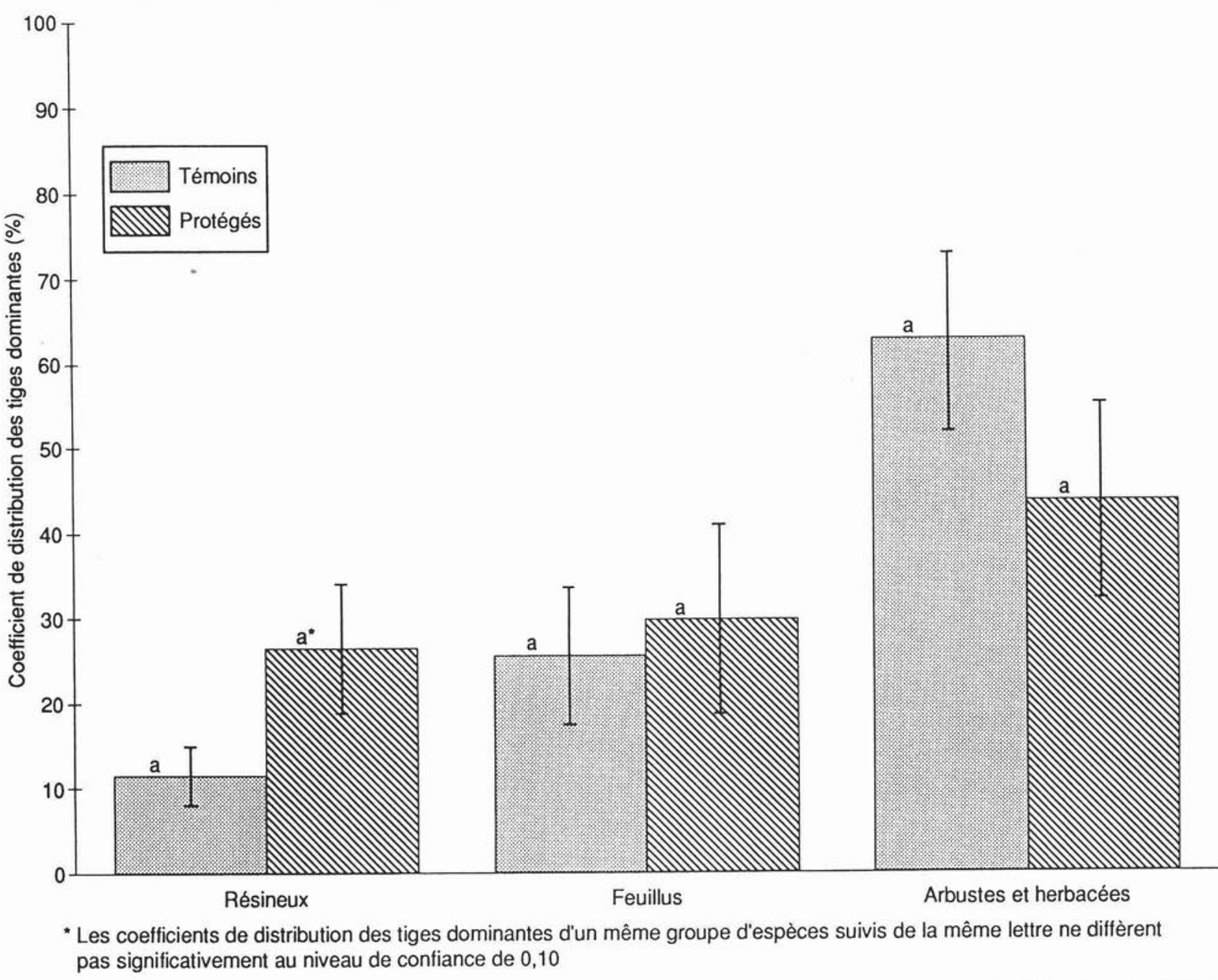

Figure 4. Coefficients de distribution des tiges dominantes 5 ans après la coupe dans les blocs témoins et protégés récoltés par abattage manuel et débusquage à câble.

pas moins que le sapin se trouve légèrement au-dessus de ces strates dans les secteurs protégés, et en-dessous dans les témoins (figure 5). Le problème d'envahissement par les arbustes et les herbacées est malgré tout commun aux deux groupes. Un remesurage serait donc nécessaire si l'on veut savoir ce qu'il adviendra de ces surfaces recouvertes d'arbustes et d'herbacées (figure 6).

Plusieurs auteurs ont observé pour le sapin une croissance lente jusqu'à l'âge de 6 ans, quelle que soit la quantité de lumière présente (Logan 1965; Zarnovican 1981; Côté et Bélanger 1991). Cette période terminée, le sapin pourrait se mettre à croître plus vite aux endroits où l'influence de la compétition se fait le moins sentir. Si c'était le cas, sa croissance serait favorisée dans les secteurs protégés où il commence à dépasser les arbustes et les herbacées.

Par ailleurs, la concurrence exercée par les feuillus apparaît plus persistante. Elle est sans doute liée à l'ouverture du couvert par la TBE avant la coupe (Osawa 1986). Dans l'échantillon étudié, si la TBE n'a pas eu d'effet déterminant sur le CDR des feuillus avant la coupe, elle pourrait néanmoins avoir favorisé la survie et la croissance avant la coupe des semis feuillus les plus âgés. Perkins et al. (1992) ont observé que la hauteur du bouleau blanc était trois fois plus importante dans des trouées que sous couvert. Pour leur part, Safford et al. (1990) rapportent une réaction du bouleau blanc proportionnelle au degré d'ouverture.

La concurrence actuellement exercée par les feuillus est susceptible de se maintenir. À l'aide de simulations sur un horizon de cinq ans, Ruel (1992) conclut à la persistance de la dominance du bouleau blanc sur le sapin. D'autres auteurs rapportent que les peuplements reconstitués à la suite d'une coupe totale comportent une plus grande proportion de feuillus (Hatcher 1964a; Frisque et al. 1978). Encore ici, un suivi sera nécessaire si l'on veut connaître la proportion des blocs que les feuillus domineront.

Malgré que la protection ait donné des avantages indéniables sur plusieurs caractéristiques de la régénération 5 ans après la coupe, les résultats ont montré une variabilité importante. Celle-ci est attribuable aux nombreux facteurs en cause: opérateurs de débusqueuses, pente, type et épaisseur des dépôts, relief, texture des sols, drainage, pierrosité, facteurs climatiques, etc. 


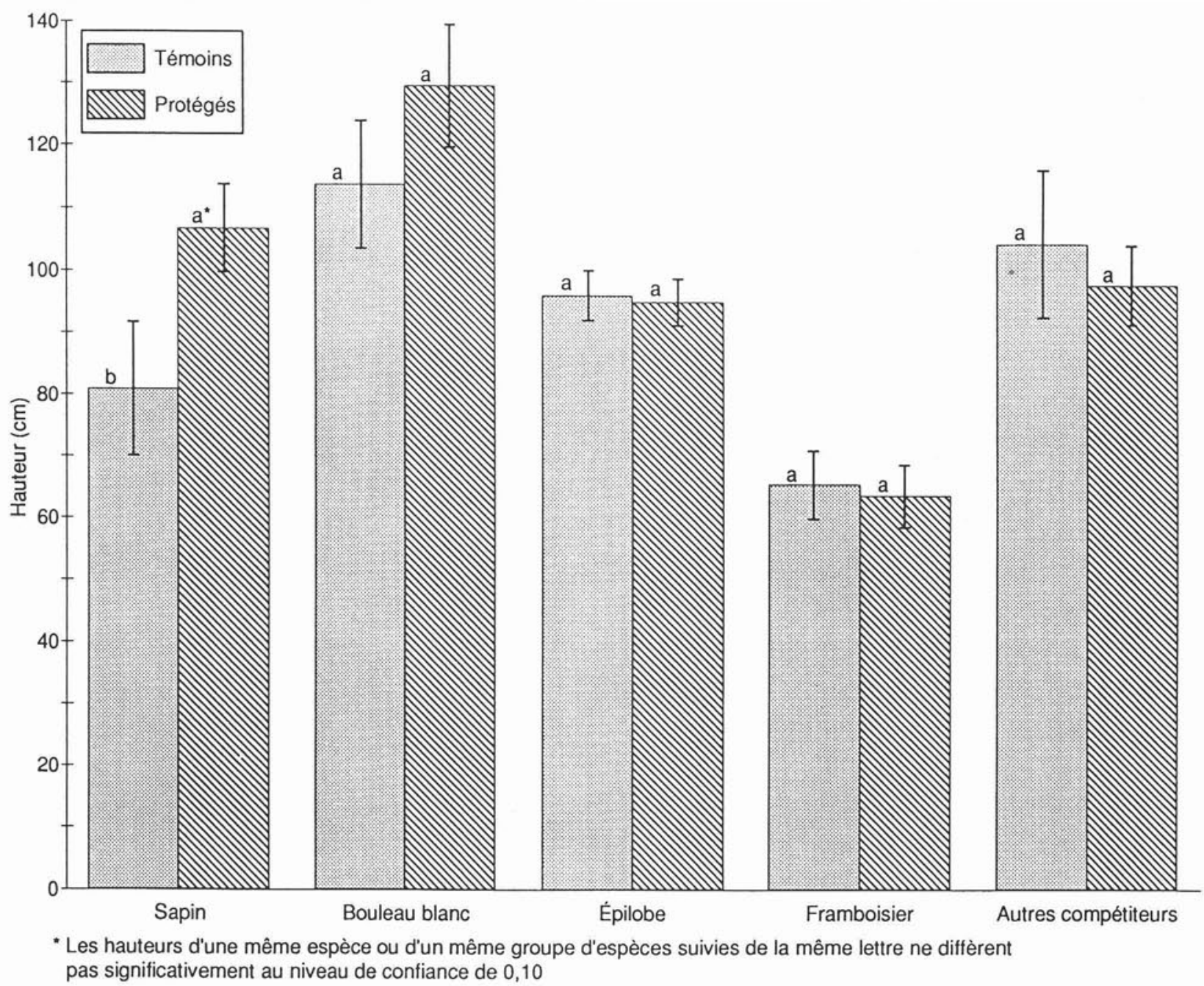

Figure 5. Hauteurs du sapin baumier, du bouleau blanc et des arbustes et herbacées 5 ans après la coupe dans les blocs témoins et protégés récoltés par abattage manuel et débusquage à câble.

\section{Conclusion}

La coupe avec protection de la régénération permet d'accroître de $25 \%$ le coefficient de distribution des résineux. C'est du moins ce qu'on observe 5 ans après la coupe. Malgré cela, ce coefficient est inférieur au seuil de $60 \%$ dans trois des cinq blocs protégés de la présente étude. Par ailleurs, les secteurs protégés présentent une régénération plus haute. Ainsi en limitant l'impact de la compétition, la coupe avec protection de la régénération permet au sapin de se hisser, en moins de cinq ans, légèrement au-dessus des arbustes et des herbacées sur environ $25 \%$ des surfaces.

Un suivi à long terme des blocs étudiés est toutefois primordial. En effet, présentement les arbustes et herbacées des blocs témoins ont un coefficient de distribution dominant de $60 \%$. Il serait donc prématuré d'émettre une conclusion finale sans connaître l'évolution de ces blocs.

\section{Remerciements}

Je tiens à remercier M. Bruno Canuel et les responsables du Service des techniques d'intervention forestière qui ont veillé à la coordination et au suivi des récoltes de données. Mes remerciements vont aussi à M. Jean-Marc Hardy qui a supervisé les travaux de coupe et la récolte des données dans l'unité de gestion de la Gaspésie. En outre, les conseils judicieux de MM. René Doucet, Jean-Claude Ruel, Fabien Caron et Pierre Bélanger, à la suite de la lecture du manuscrit, ont hautement été appréciés. Enfin, je remercie M. Louis Blais pour son aide relative aux questions statistiques, Mmes Sylvie Bourassa et Nathalie Samson pour la dactylographie et M. Lévis Beaulieu pour la confection des graphiques.

\section{Bibliographie}

Canuel, B., 1987. Guide d'utilisation de la coupe avec protection de la régénération (abattage manuel). Min. de l'Énergie et des Ressources du Québec, Service des traitements sylvicoles. 16 p.

Côté, S. et L. Bélanger. 1991. Variations de la régénération préétablie dans les sapinières boréales en fonction de leurs caractéristiques écologiques. Can. J. For. Res. 21: 1779-1795.

Deschênes, C. 1983. Normes d'inventaire relatives à l'évolution de la régénération naturelle en fonction des procédés et des modes 


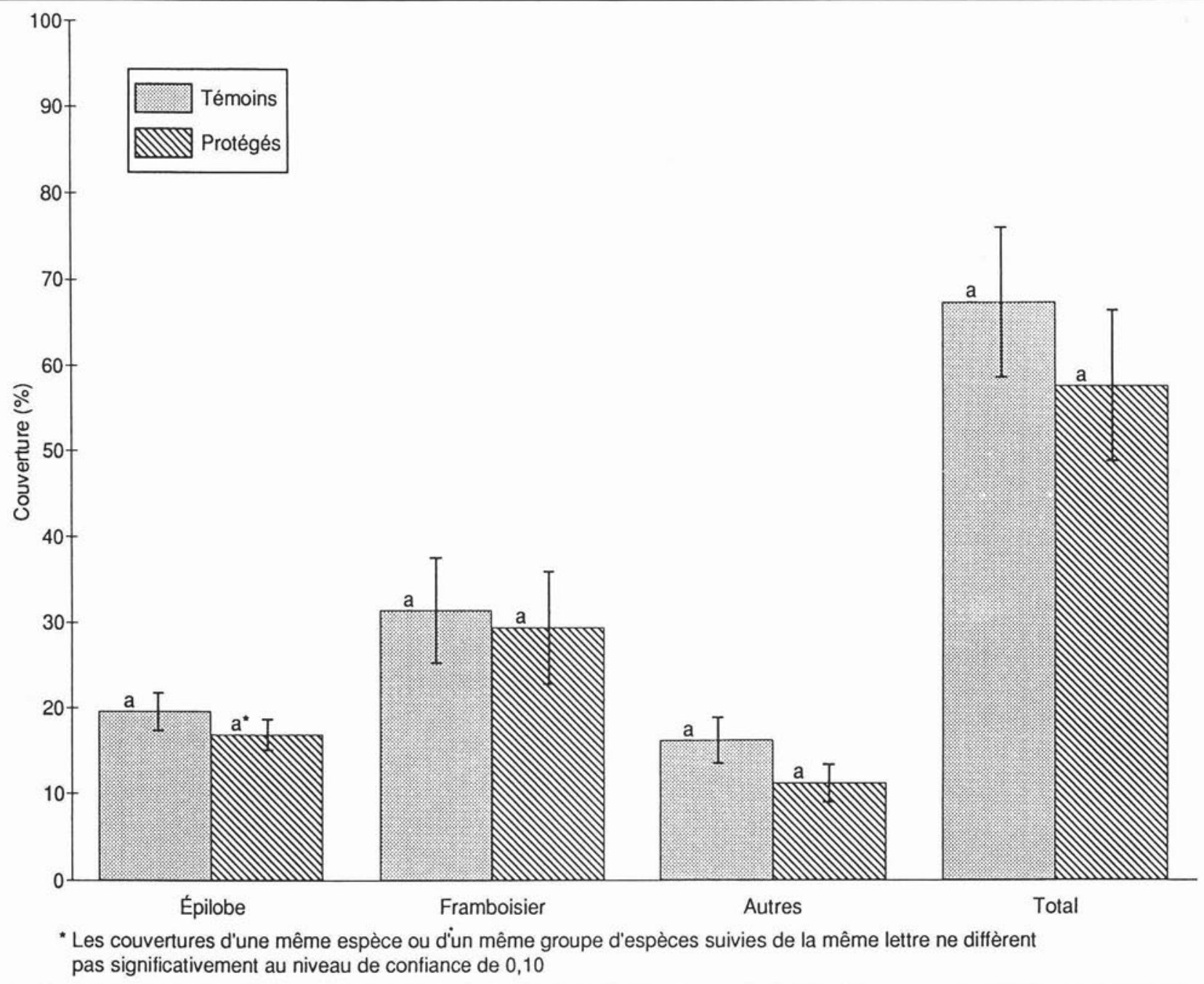

Figure 6. Couverture des principaux arbustes et herbacées 5 ans après la coupe dans les blocs témoins et protégés récoltés abattage manuel et débusquage à câble.

de récolte. Min. de l'Énergie et des Ressources du Québec, Service de la technologie et des normes d'exploitation. $37 \mathrm{p}$.

Deschênes, C. 1991. Régénération naturelle 5 ans, 9 ans et 14 ans après coupe sur sites humides en Abitibi. Min. des Forêts du Québec, Service des interventions forestières. $65 \mathrm{p}$.

Doucet, R. 1988. La régénération préétablie dans les peuplements forestiers naturels au Québec. For. Chron. 64: 116-120.

Frank, R.M. et E.L. Putnam. 1972. Seedling survival in sprucefir after mechanical tree harvesting in strips. USDA For. Serv., Northeast. For. Exper. Sta., Res. Pap. NE-224. 16 p.

Fraser, J.W. 1976. Viability of black spruce seed in or on boreal seedbed. For. Chron. 52: 229-231.

Frisque, G., G.F. Weetman et E. Clemmer. 1978. Analyse, 10 ans après coupe de bois à pâte, des problèmes de régénération dans l'Est du Canada. Institut canadien de recherche en génie forestier. Rapport technique $\mathrm{n}^{\circ}$ RT-23. $67 \mathrm{p}$.

Fye, R.E. et J.B. Thomas. 1963. Regeneration of balsam fir and spruce about fifteen years following release by spruce budworm attack. For. Chron. 39: 385-397.

Godin, B. 1986. Forecasting cutover response in New Brunswick: a guide for silvicultural decison making. New Brunswick Dept. Forest, Mines and Energy. $71 \mathrm{p}$.

Hardy, J.-M. 1988. Évaluation de quelques expériences de protection de la régénération naturelle lors de la récolte. Min. de
l'Énergie et des Ressources du Québec. Bureau régional de la Gaspésie. 19 p.

Hatcher, R.J. 1964a. Croissance du sapin baumier après une coupe rase dans le Québec. Canada, Min. Nord Can. et Ress. nation., Div. rech. sylv., Mém. tech. ${ }^{\circ} 87.24$ p.

Hatcher, R.J. 1964b. Spruce budworm damage to balsam fir in immature stands Québec. For. Chron. 40: 372-383.

Heidersdorf, E. et M. Ryans. 1986. Essais conjoints FERIC/MER des pneus à haute flottaison dans les régions du Saguenay-Lac SaintJean et d'Abitibi-Témiscamingue en 1984. Inst. can. rech. en génie for., Rapp. tech. $\mathrm{n}^{\circ}$ RT-64. 53 p.

Huot, M. et J.-C. Ruel. 1990. Répercussions d'une épidémie de tordeuse des bourgeons de l'épinette sur le renouvellement naturel des sapinières. Annales de l'ACFAS 58: 311.

Lanteigne, L.J. et I.R. Methven. 1986. La réponse, après la coupe, d'un peuplement d'épinettes et de sapins du centre du Nouveau-Brunswick. Recueill de communications présentées au $67^{\mathrm{e}}$ congrès annuel de la Division des bois et forêts de l'Association canadienne des producteurs de pâtes et papiers. Montréal, 28-29 janvier 1986. F35-F38.

Logan, K.T. 1965. Growth of tree seedlings as affected by light intensity. I. White birch, yellow birch, sugar maple and silver maple. Can. Dept. For. Publ. n ${ }^{\circ} 1121.15$ p.

Maclean, D.A. 1984. Effects of spruce budworm outbreaks on 
the productivity and stability of balsam fir forests. For. Chron. 60 : 273-279.

Marks, P.L. 1974. The role of pin cherry (Prunus pensylvanica L.) in the maintenance of stability in northern hardwood ecosystems. Ecol. Monogr. 44: 73-88.

Marquis, D.A., D.S. Solomon et J.C. Bjorkbom. 1969. A silvicultural guide for paper birch in the Northeast. USDA For. Serv. Res. Northeast For. Exp. Sta. Paper NE-130.

Mott, D.G. 1974. The consequences of applying no control to epidemic spruce budworm in Eastern spruce-fir. Dans: Proc. Symp. on the Spruce Budworm, Alexandria, Virginia. USDA For. Serv. Misc. Pub. 1327: 67-72.

Ohman, L.F. et R.R. Ream. 1971. Wilderness ecology. Virgin plant communities of the Boundary Waters Canoe Area. USDA. For. Serv. North Central For. Exp. Stn. Res. Pap. NC-63. 55 p. Osawa, A. 1986. Patch dynamics of spruce-fir forests during a spruce budworm outbreak in Maine. Ph.D. Thesis, Yale University. $128 \mathrm{p}$.

Perkins, T.D., R.M. Klein, G.J. Badger et M.J. Easter. 1992. Spruce-fir decline and gap dynamics on Camels Hump, Vermont. Can. J. For. Res. 22: 413-422.

Pominville, P. et R. Doucet. 1992. Coefficients de distribution de la régénération nécessaires au maintien de la production des peuplements de pin gris, d'épinette noire et de sapin baumier. Min. des Forêts du Québec, Direction de la recherche. Note de recherche forestière $\mathrm{n}^{\circ} 48.16 \mathrm{p}$.

Potvin, F. 1980. Short-term impact of a spruce budworm outbreak on a deer wintering area. Can. J. For. Res. 10: 559-563.

Québec, 1984. Normes d'inventaire forestier. Min. de l'Énergie et des ressources, Direction générale des forêts, Service de l'inventaire forestier. ISBN2-551-06406-6. $177 \mathrm{p}$.

Québec, 1991. Loi sur les forêts (L.R.Q., chap. F-4.1). Bibli. Nat. du Québec. Publ.: ISBN2-551-14773-5. Éditeur officiel du Québec. Ruel, J.-C. 1987. Effect de différents procédés de récolte de la matière ligneuse sur la régénération préétablie. Min. de l'Énergie et des Ressources, Direction de la recherche et du développement. Rapport interne $\mathrm{n}^{\circ} 282$.
Ruel, J.-C. 1989a. Régénération des interbandes dans un système de coupes par bandes de peuplements d'épinette noire. For. Chron. 65: $372-376$

Ruel, J.-C. 1989b. Importance de la régénération préexistante dans les forêts publiques du Québec. Ann. Sci. For. 46: 345-359.

Ruel, J.-C. 1990. Advance growth abundance and regeneration patterns after clearcutting in Québec. Proceedings of the conference on natural regeneration management. Forêts Canada, Région des Maritimes. Hugh John Fleming Forestry Centre, Fredericton, N.B.: $115-131$

Ruel, J.-C. 1992. Impact de la compétition exercée par le framboisier (Rubus idaeus L.) et les feuillus de lumière sur la croissance du sapin (Abies balsamea (L.) Mill.) en régénération. Can. J. For. Res. 22: 1408-1416.

Safford, L.O., J.C. Bjorkbam et J.C. Zasada. 1990. Betula papyrifera Marsh. Silvics of North America. USDA. For. Serv. Res. Dept. Agr. Hand. 654. p. 158-171.

Schoenike, R.E. et H.L. Hansen. 1954. Viability of black spruce seed in four-year-old logging slash. Minnesota For. Notes No. 29. 2 p.

Thibault, M. 1985. Les régions écologiques du Québec méridional. Québec: Min. de l'Énergie et des Ressources, Serv. de la rech. et Serv. de la cartographie. Carte couleurs 1: 1250000.

Tremblay, M., J. Martel, R. Doucet et H. Bolgahri. 1987. Régénération naturelle. Opérations forestières et de scieries, nov.déc.: 22-24.

Wall, R.E. 1983. Early stand development after clear-cutting on the Cape Breton highlands. Envir. Canada, For. Serv., Inf. Rep. M-X-143. $16 \mathrm{p}$.

Webber, B., J.T. Arnott, G.F. Weetman et G.C.R. Cromme. 1968. Advance growth destruction, slash coverage and ground conditions in logging operations in eastern Canada. Pulp. Pap. Res. Inst. Can., Woodl. Rep. No. 8. 109 p.

Zarnovican, R. 1981. À propos de l'âge du sapin baumier et de sa détermination. Can. J. For. Res. 11: 805-811. 